\title{
22. A TECHNIQUE FOR OBTAINING PORE-WATER CHEMICAL COMPOSITION FROM INDURATED AND HYDROTHERMALLY ALTERED SEDIMENT AND BASALT: THE GROUND ROCK INTERSTITIAL NORMATIVE DETERMINATION (GRIND) ${ }^{1}$
}

\author{
C. Geoffrey Wheat, ${ }^{2}$ Jacques Boulegue, ${ }^{3}$ and Michael J. Mottl ${ }^{2}$
}

\begin{abstract}
During Ocean Drilling Program Leg 139 at Middle Valley, Juan de Fuca Ridge, we recovered indurated sediment and hydrothermally altered diabases and gabbros from an active hydrothermal reservoir at Sites 857 and 858 . Because few of these samples yielded pore water upon squeezing even at the maximum pressure afforded by the hydraulic presses aboard JOIDES Resolution, we developed the GRIND technique (ground rock interstitial normative determination). The GRIND technique consists of fragmenting a freshly collected sample, grinding the sample with distilled water in a ball mill, squeezing the ground mixture, and analyzing the effluent.

Concentrations of $\mathrm{Mg}, \mathrm{Ca}, \mathrm{Na}, \mathrm{K}, \mathrm{Rb}$, and, from Site 857 only, $\mathrm{Sr}$, measured in GRIND samples are comparable to measurements of squeezed samples, in contrast to alkalinity, sulfate, dissolved silica, $\mathrm{Ba}, \mathrm{Mn}, \mathrm{Li}$, and $\mathrm{B}$, which differ substantially between the two methods. GRIND samples with chlorinity greater than about $100 \mathrm{mmol} / \mathrm{kg}$ have only minimal artifacts caused by ion exchange. Ion exchange in more diluted pore-water samples causes an increase in $\mathrm{Na}$ and a decrease in $\mathrm{Mg}, \mathrm{Ca}$, and $\mathrm{Sr}$. The GRIND method could be an invaluable tool for estimating the chemical composition of pore waters in basement.
\end{abstract}

\section{INTRODUCTION}

Knowledge of the composition of pore waters throughout a sediment column is an important tool for understanding the physical, chemical, biological, and geological processes that have currently or recently influenced diagenesis of the sediment and alteration in basement. For example, changes in the pore-water concentrations of dissolved oxygen, nitrate, sulfate, ammonium, manganese, iron, alkalinity, and phosphate provide a measure of biologically mediated reactions. Concentrations of other ions such as dissolved silica, lithium, potassium, rubidium, magnesium, and calcium change from bottom seawater values to values in equilibrium with sediment and basement. These data are useful in assessing rates of inorganic diagenesis. Pore-water profiles of conservative ions such as chlorinity, and in certain circumstances, magnesium, can also be used as a tracer for pore-water flow.

Because the chemical composition of pore waters is so useful for determining present-day processes, much care is taken to recover pore-water samples that are not affected by sampling procedures. Several in-situ and shipboard techniques have been developed to extract pore water from sediment. In-situ techniques rely on positioning an entry port into sediment and then drawing pore water into a reservoir through a filter (e.g., Barnes, 1988). Samples retrieved using in-situ techniques are not affected by artifacts caused by changes in temperature or pressure, but these in-situ tools are expensive and can only be used in soft sediment that will not crack and allow bottom water to flow and contaminate the sample. Shipboard techniques to obtain pore water have relied on squeezing sediment through a filter into a container (e.g., Manheim and Sayles, 1974), or centrifuging a sediment sample. Samples obtained using these methods are subject to artifacts caused by sample handling and changes in temperature, pressure, and oxidation state (de Lange et al., 1992). Squeezing typically produces a

\footnotetext{
${ }^{1}$ Mottl, M.J., Davis, E.E., Fisher, A.T., and Slack, J.F. (Eds.), 1994. Proc, ODP, Sci. Results, 139: College Station, TX (Ocean Drilling Program).

${ }_{3}^{2}$ SOEST, 1000 Pope Road, University of Hawaii, Honolulu, HI 96822, U.S.A. France.
}

greater volume of pore water than centrifugation; however, little to no water can be extracted from the most lithified sediment or hydrothermally altered basalt or gabbro by either technique.

During Ocean Drilling Program (ODP) Leg 139 in Middle Valley, Juan de Fuca Ridge, we recovered indurated sediment and hydrothermally altered diabases and gabbros from Sites 857 and 858 . We tried to recover pore water using a Carver hydraulic press (Manheim and Sayles, 1974) with a maximum pressure of $2.8 \mathrm{MPa}$. Many of these samples did not yield any pore water even after $12 \mathrm{hr}$ at the maximum safe pressure. These samples comprise over half of the sediment column at Site 857, half of Hole 858F, and all of Hole 858G. Site 857 was positioned to drill into the hydrothermal fluid reservoir that is thought to supply fluids to active hydrothermal vents $1.5 \mathrm{~km}$ to the north, where Site 858 is located. Holes $858 \mathrm{~F}$ and $858 \mathrm{G}$ are located within $50 \mathrm{~m}$ of active hydrothermal vents where effluent from these vents reaches temperatures of $276^{\circ} \mathrm{C}$ (Butterfield et al., this volume), about the temperature at $500 \mathrm{mbsf}$ in Site 857 and at $50 \mathrm{mbsf}$ in Holes 858F and 858G (Davis, Mottl, Fisher, et al., 1992). An important focus of Leg 139 was to understand the chemical processes that take place in these holes by examining the pore-water chemical composition, physical properties, mineralogy, and geochemistry of rocks that make up the hydrothermal reservoir.

Because most of the sediment and rock samples did not yield any pore water upon squeezing, we modified a method presented by Cranston (1991), herein referred to as the GRIND technique (ground rock interstitial normative determination), to obtain information on the composition of pore water from indurated sediment and hydrothermally altered basalt and gabbro. In this paper we present a comparison of the chemical composition of pore water obtained by squeezing sediment using a titanium squeezer (Manheim and Sayles, 1974) and by using the GRIND technique on samples from Sites 857 and 858 . This comparison provides the data from which we determine probable reactions that occur during the GRIND procedure that result in changes in the chemical composition of the pore waters. Much of the paper focuses on the potential sources for chemical change during sample handling and the inherent problems associated with the GRIND technique, as it is important for future geochemists involved in ODP to understand the limitations of the technique, and of the conclusions one can draw from data obtained using the technique. 


\section{ANALYTICAL METHODS}

The general idea of the GRIND technique is to fragment a freshly collected sample, then grind the sample with distilled water in a ball mill. The ground mixture is then squeezed and the effluent is analyzed. No attempt is made to conduct the procedure at in-situ temperature or pressure because of the lack of a ball mill that can be heated to $275^{\circ} \mathrm{C}$ at 300 bars, and because these elevated temperatures and pressures enhance chemical reactions that occur as a result of dilution of the sample with distilled water. No attempt is made to process the samples at in-situ oxidation state because few of the chemical species of interest are affected by changes in the oxidation state and because samples were exposed to dissolved and atmospheric oxygen during coring and retrieval.

Whole-round samples of indurated sediment and hydrothermally altered basalt were processed as soon as possible after recovery. Typically one subsample was broken off and squeezed while another was subjected to the GRIND technique. Samples subjected to the GRIND technique were first blotted with paper towels to remove seawater that came in contact with the sample during drilling and retrieval. If these samples were not immediately processed, they were wrapped tightly in parafilm and stored in a refrigerator for at most one day.

The GRIND process consists of placing the sample in double plastic bags and fragmenting it using a hammer, until all of the sample is $<1 \mathrm{~cm}$ in size. About $35 \mathrm{~g}$ of the sample is then weighed and placed in a ball mill. A measured volume of deionized distilled water is added to the ball mill, then the mixture is ground and squeezed to extract the pore water/distilled water mixture. The optimal volume of water that must be added to the ball mill is less than the porosity multiplied by the mass of the sample. We generally added $5 \mathrm{~mL}$ of deionized distilled water to $35 \mathrm{~g}$ of sediment, about a one-to-one ratio between distilled water and pore water. Excess distilled water results in a ground slurry that is difficult to transfer to the squeezer and effectively extract the water. A lesser volume of distilled water may not produce pore water upon squeezing or may produce too small a volume to be of use. The optimal grinding time is $15 \mathrm{~min}$. Shorter periods of time did not adequately crush the sample, and longer periods may enhance reactions between the sample and distilled water.

Pore waters obtained using the GRIND technique are subject to the same analytical procedures as squeezed samples. The analytical methods used to determine the composition of the pore waters are described by the Shipboard Scientific Party (1992) and Wheat and Mottl (this volume) and consist of standard colorimetric, potentiometric, and spectrometric techniques (Tables 1 and 2).

The GRIND method is similar to the one presented by Cranston (1991) in that deionized distilled water is added to a sediment sample. However, we did not degas the distilled water or handle the samples in an oxygen-free environment at in-situ temperatures. The main difference between the two techniques is that Cranston's method is designed for high-resolution sediment sampling using small sample sizes when either sediment availability or manpower for squeezing is limiting, whereas the GRIND method is designed to provide some pore-water chemical data from lithified sediments and hydrothermally altered basalts that do not yield pore water when squeezed at pressures to $2.8 \mathrm{MPa}$ for over $12 \mathrm{hr}$.

\section{RESULTS AND DISCUSSION}

In the following sections we present the data and discuss sources of pore-water contamination during the GRIND technique. Although most of this section focuses on the problems associated with the GRIND technique, the extent of reaction was found to be minimal for some chemical species, indicating that the GRIND technique can provide useful data for the composition of pore water in indurated and hydrothermally altered sediments and rocks.

There are several potential sources for error using the GRIND method. Sample handling occurs at room temperature and pressure, both of which are much less than in-situ values. Adding distilled water during the grinding stage alters the ionic strength of the pore water. Because of these procedures, ion-exchange or dissolution/precipitation reactions may result. Because the sample is exposed to atmospheric oxygen and dissolved oxygen in the added distilled water, redox reactions may alter the pore-water composition. For example, the oxidation of reduced dissolved iron in the pore water results in an oxidized form that readily precipitates, thus lowering the concentration of dissolved iron. Oxidation reactions are reduced if all of the work is done with deoxygenated water in a nitrogen-filled glove bag.

In the following sections, we compare the chemical composition of pore waters extracted using the GRIND technique with those obtained from squeezing. Profiles of the concentration in pore water vs. depth are presented for the major and minor ions in seawater based on GRIND and squeezed samples in which the GRIND data are adjusted to account for an estimated pore-water chlorinity of 590 $\mathrm{mmol} / \mathrm{kg}$ at Site 857 and $565 \mathrm{mmol} / \mathrm{kg}$ at Site 858 . These plots are useful in assessing trends in the data, the range of values obtained using the GRIND and squeezed techniques, and potential sources of contamination during the GRIND technique. We also include plots of the ratio of dissolved elements to chlorinity for GRIND vs. squeezed samples. This allows for a direct comparison of the two methods; however, only 20 of the 60 GRIND samples have corresponding squeezed samples and 3 of these GRIND samples are duplicates or triplicates (Table 3). To examine the effect of dilution on the GRIND samples, we present chemical profiles in which the most diluted GRIND samples are highlighted. We also present plots of the ion concentration divided by chlorinity, to normalize for the extent of dilution, vs. the chlorinity of the sample, which represents the extent of dilution.

\section{Major Ions}

Dilution of the pore-water samples during the GRIND method causes a change in the ionic strength and the concentrations of the ions. Assuming the pore water is originally in equilibrium with its surroundings, dilution can result in disequilibrium. If the kinetics of reaction are faster than the time required for grinding and squeezing the sample, the chlorinity-adjusted chemical composition of pore water using the GRIND technique will be different from that obtained by squeezing. Cranston (1991) states that dilution of pore water results in exchange reactions that decrease the concentrations of divalent cations in pore waters while increasing the concentration of monovalent cations.

Depth profiles of dissolved species provide insight into the diffusive, advective, and reactive processes that presently exist, but the effects of sample handling must be understood. Dilution of the sample during the GRIND process generally increases the concentration of dissolved $\mathrm{Na}$ (Fig. 1). The most diluted GRIND samples $(<100 \mathrm{mmol}$ $\mathrm{Cl} / \mathrm{kg}$ ) are highlighted in depth profiles and the concentration of $\mathrm{Na}$ in these samples is systematically greater than that in neighboring squeezed and more concentrated GRIND samples (Figs. 1A and 1B). Likewise, a comparison of GRIND and squeezed samples from the same depth interval reveals that the ratio of $\mathrm{Na}$ to chlorinity in the GRIND samples is generally greater than that in the squeezed samples (Fig. 1C), implying that ion exchange increases the concentration of $\mathrm{Na}$ in the more diluted pore waters obtained from the GRIND process. Lastly, a plot of the ratio of $\mathrm{Na}$ to chlorinity vs. chlorinity shows an increase in $\mathrm{Na} / \mathrm{Cl}$ with decreasing chlorinity (Fig. 1D).

Note, however, that trends in ion/chlorinity vs. chlorinity plots can result from ion exchange during sample handling as well as from changes in the ion concentration with depth. Clearly, as presented above, ion exchange during sample handling influences the concentration of dissolved $\mathrm{Na}$ in GRIND samples (Figs. 1A, 1B, and 1C). Some of the trend in Figure 1D results from a general increase in $\mathrm{Na}$ with depth that is real, but which corresponds with a general decrease in chlorinity that is related to a decrease in porosity. In Figure 2 we 
Table 1. The composition of water extracted from lithified sediment and hydrothermally altered basalt from Site 857 using the GRIND technique.

\begin{tabular}{|c|c|c|c|c|c|c|c|c|c|c|c|c|c|c|c|c|c|c|c|}
\hline Sample & $\begin{array}{l}\text { Core, section, } \\
\text { interval }(\mathrm{cm})\end{array}$ & $\begin{array}{l}\text { Depth } \\
\text { (mbsf) }\end{array}$ & $\begin{array}{c}\text { Volume } \\
(\mathrm{mL})\end{array}$ & $\begin{array}{l}\text { Squeeze } \\
\text { pressure } \\
\text { (psi) }\end{array}$ & $\mathrm{pH}$ & $\begin{array}{c}\text { Alkalinity } \\
\text { (meq/kg) }\end{array}$ & $\begin{array}{l}\text { Chlorinity } \\
(\mathrm{mmol} / \mathrm{kg})\end{array}$ & $\begin{array}{c}\text { Sulfate } \\
(\mathrm{mmol} / \mathrm{kg})\end{array}$ & $\begin{array}{c}\mathrm{Na} \\
(\mathrm{mmol} / \mathrm{kg})\end{array}$ & $\underset{(\mathrm{mmol} / \mathrm{kg})}{\mathrm{K}}$ & $\underset{(\mathrm{mmol} / \mathrm{kg})}{\mathrm{Mg}}$ & $\begin{array}{c}\mathrm{Ca} \\
(\mathrm{mmol} / \mathrm{kg})\end{array}$ & $\begin{array}{c}\mathrm{Si} \\
(\mu \mathrm{mol} / \mathrm{kg})\end{array}$ & $\begin{array}{c}\mathrm{Mn} \\
(\mu \mathrm{mol} / \mathrm{kg})\end{array}$ & $\begin{array}{c}\mathrm{Sr} \\
(\mu \mathrm{mol} / / \mathrm{kg})\end{array}$ & $\begin{array}{c}\mathrm{Ba} \\
(\mu \mathrm{mol} / / \mathrm{kg})\end{array}$ & $\begin{array}{c}\text { B } \\
(\mu \mathrm{mol} / \mathrm{kg})\end{array}$ & $\begin{array}{c}\mathrm{Li} \\
(\mu \mathrm{mol} / \mathrm{kg})\end{array}$ & $\begin{array}{c}\mathrm{Rb} \\
(\mu \mathrm{mol} / \mathrm{kg})\end{array}$ \\
\hline \multicolumn{20}{|c|}{$139-857 \mathrm{C}-$} \\
\hline G-5 & $37 \mathrm{R}-1,140-150$ & 352.25 & 5.5 & 25000 & 7.61 & 0.590 & 284.3 & 2.96 & 190 & 15.4 & 6.11 & 36.19 & 167 & 9.25 & 179.0 & 10.3 & 300 & 187 & 4.66 \\
\hline $\mathrm{G}-2$ & $46 \mathrm{R}-2,0-15$ & 400.58 & 8.0 & 5000 & 7.31 & 0.478 & 170.9 & 0.83 & 123 & 6.8 & 3.31 & 18.42 & 133 & 4.03 & 64.7 & 10.5 & 190 & 95 & 2.95 \\
\hline G-1 & $46 \mathrm{R}-2,0-15$ & 400.58 & 7.5 & 2000 & 7.91 & 0.581 & 131.3 & 0.78 & 96 & 5.0 & 1.33 & 14.83 & 133 & 2.02 & 47.0 & 8.2 & 457 & 72 & 2.26 \\
\hline$G-3$ & $46 \mathrm{R}-2,0-15$ & 400.58 & 3.0 & 10000 & & & 273.2 & 1.48 & 192 & 10.1 & 4.73 & 32.47 & 170 & & & & & & \\
\hline G-7 & $47 \mathrm{R}-1,135-150$ & 405.43 & 3.9 & 30000 & 7.52 & 0.526 & 273.2 & 0.90 & 187 & 10.5 & 3.83 & 35.16 & 163 & 6.29 & 115.0 & 21.3 & 237 & 151 & 3.70 \\
\hline $\mathrm{G}-4$ & $48 \mathrm{R}-1,132-147$ & 410.10 & 4.2 & 25000 & 7.71 & 0.557 & 266.0 & 1.38 & 183 & 12.0 & 4.19 & 32.61 & 153 & 4.62 & 104.0 & 11.6 & 235 & 126 & 3.53 \\
\hline G-6 & $49 \mathrm{R}-1,135-150$ & 415.13 & 4.2 & 25000 & 7.73 & 0.755 & 294.5 & 1.20 & 204 & 11.7 & 4.96 & 35.86 & 175 & 6.62 & 121.0 & 15.8 & 317 & 149 & 5.30 \\
\hline G-21 & $49 \mathrm{R}-1,135-150$ & 415.13 & 5.5 & 15000 & 7.63 & 1.483 & 258.7 & 7.55 & 179 & 12.0 & 6.86 & 35.11 & 178 & 15.3 & 94.0 & 2.9 & 290 & 63 & 3.23 \\
\hline G-8 & $55 \mathrm{R}-1,36-38$ & 442.37 & 5.2 & 12000 & 7.80 & 0.556 & 290.6 & 2.08 & 207 & 9.7 & 8.37 & 30.54 & 84 & 5.10 & 110.0 & 8.8 & 336 & 130 & 6.69 \\
\hline G-9 & $56 \mathrm{R}-1,10-14$ & 446.62 & 4.5 & 20000 & 7.69 & 0.640 & 268.4 & 1.70 & 191 & 9.1 & 5.83 & 30.29 & 100 & 7.48 & 103.0 & 10.4 & 255 & 96 & 3.44 \\
\hline G-10 & $57 \mathrm{R}-1,0-20$ & 451.80 & 4.2 & 20000 & 7.82 & 0.622 & 281.9 & 2.62 & 204 & 9.3 & 8.04 & 28.80 & 100 & 3.50 & 100.0 & 8.1 & 296 & 115 & 8.09 \\
\hline G-11 & $58 \mathrm{R}-\mathrm{CC}, 0-22$ & 471.00 & 4.6 & 30000 & 7.72 & 0.786 & 275.2 & 2.37 & 197 & 9.4 & 7.68 & 29.53 & 112 & 7.53 & 97.6 & 7.5 & 223 & 90 & 4.06 \\
\hline G-19 & $62 \mathrm{R}-1,142-150$ & 501.46 & 5.0 & 25000 & 7.34 & 7.988 & 196.2 & 2.08 & 144 & 6.0 & 2.61 & 26.67 & 178 & 0.00 & 72.6 & 5.8 & 73 & 23 & 4.86 \\
\hline $\mathrm{G}-12$ & $66 \mathrm{R}-1,4-9$ & 538.87 & 4.5 & 30000 & 7.90 & 0.952 & 251.1 & 2.78 & 189 & 6.2 & 8.06 & 23.04 & 123 & 2.93 & 82.6 & 5.3 & 194 & 55 & 5.66 \\
\hline \multicolumn{20}{|c|}{ 139-857D- } \\
\hline G-1 & $1 \mathrm{R}-2,5-12$ & 583.09 & 3.0 & 10000 & 7.29 & 0.830 & 216.1 & 0.75 & 158 & 5.2 & 7.05 & 20.69 & 90 & 3.43 & 64.2 & 6.5 & & & \\
\hline G-3 & $3 \mathrm{R}-2,0-7$ & 600.84 & 3.0 & 30000 & 7.42 & 0.607 & 99.1 & 0.38 & 75 & 2.0 & 1.60 & 10.12 & 83 & 0.32 & 21.2 & 4.0 & 20 & 2.00 & 1.68 \\
\hline $\mathrm{G}-2$ & $4 \mathrm{R}-2,68-72$ & 611.10 & 2.5 & 25000 & & & 113.1 & 0.17 & 87 & 2.2 & 2.34 & 9.98 & 72 & 2.11 & 27.8 & 1.7 & 33 & 3.47 & 1.74 \\
\hline $\mathrm{G}-4$ & $9 \mathrm{R}-1,33-36$ & 657.25 & 2.5 & 35000 & & & 58.5 & 0.00 & 46 & 1.0 & 0.75 & 5.00 & 76 & 0.21 & 9.4 & 2.1 & 22 & 1.17 & \\
\hline $\mathrm{G}-6$ & $10 \mathrm{R}-1,19-21$ & 666.82 & 4.0 & 10000 & 7.33 & 0.584 & 238.4 & 0.74 & 175 & 6.2 & 6.05 & 23.42 & 82 & 2.97 & 74.0 & 5.3 & 116 & 23.12 & 5.74 \\
\hline G-9 & $11 \mathrm{R}-1,20-23$ & 676.42 & 3.5 & 30000 & 7.34 & 0.649 & 191.9 & 0.48 & 143 & 4.8 & 4.73 & 18.20 & 83 & 1.81 & 59.8 & 5.9 & 72 & & 3.63 \\
\hline G-5 & $12 \mathrm{R}-1,16-20$ & 686.08 & 4.0 & 12000 & 7.26 & 0.631 & 160.1 & 0.52 & 120 & 4.3 & 2.35 & 16.46 & 96 & 1.72 & 47.0 & 6.1 & 58 & & \\
\hline \multirow{2}{*}{ G-7 } & $12 \mathrm{R}-1,16-20$ & 686.08 & 4.0 & 15000 & 7.20 & 0.436 & 157.0 & 0.20 & 109 & 4.6 & 2.40 & 19.44 & 80 & 2.10 & 54.3 & 7.1 & 65 & 33.1 & 3.78 \\
\hline & & & & & & & & & & & & & & 0.44 & 82.4 & 12.2 & 62 & 80.8 & 1.36 \\
\hline G-11 & $15 \mathrm{R}-1,10-14$ & 714.92 & 4.0 & 35000 & 7.34 & 0.679 & 187.7 & 0.89 & 142 & 5.0 & 4.70 & 17.09 & 98 & 2.15 & 44.2 & 2.8 & 73 & 12.3 & \\
\hline G-8 & $16 \mathrm{R}-1,8-11$ & 724.60 & 3.5 & 25000 & & & 165.0 & 0.22 & 124 & 3.7 & 3.21 & 15.62 & 82 & 1.67 & 50.2 & 3.1 & 48 & 7.20 & 3.12 \\
\hline $\mathrm{G}-10$ & $17 R-2,109-111$ & 736.50 & 3.0 & 35000 & & & 141.5 & 0.12 & 111 & 2.8 & 2.76 & 11.28 & 71 & 0.94 & 34.3 & 1.7 & 42 & 2.28 & 1.78 \\
\hline $\mathrm{G}-12$ & $19 R-1,18-19$ & 752.99 & 3.0 & 25000 & & & 56.6 & 0.41 & 47 & 1.6 & 0.39 & 4.18 & 244 & 0.09 & 10.0 & 0.8 & 25 & 6.44 & 1.28 \\
\hline G-13 & $20 \mathrm{R}-1,90-92$ & 763.41 & 2.8 & 25000 & & & 40.3 & 0.35 & 34 & $\begin{array}{l}1.0 \\
1.2\end{array}$ & 0.18 & 2.90 & 278 & 0.09 & 6.3 & 0.4 & 20 & 0.44 & 1.20 \\
\hline G-14 & 2 IR $-2,30-34$ & 774.02 & 3.5 & 15000 & 7.50 & 1.154 & 230.9 & 1.31 & 175 & 5.0 & 8.70 & 18.48 & 59 & 0.36 & 12.6 & 0.8 & 24 & 15.2 & 4.31 \\
\hline G-15 & $23 \mathrm{R}-1,10-11$ & 791.61 & 2.5 & 35000 & & & 46.5 & 0.09 & 37 & 1.1 & 0.30 & 4.12 & 98 & 0.09 & 5.4 & 1.2 & 21 & 0.72 & \\
\hline G-16 & $24 \mathrm{R}-1,66-69$ & 801.88 & 3.0 & 30000 & 7.26 & 0.644 & 146.3 & 0.16 & 112 & 3.3 & 3.54 & 12.62 & 69 & 0.98 & 41.5 & 2.3 & 37 & 1.66 & 2.73 \\
\hline G-17 & $25 \mathrm{R}-1,41-42$ & 811.22 & 3.0 & 35000 & 1.20 & $0.0+4$ & 44.6 & 0.15 & 36 & 0.9 & 0.46 & 3.70 & 126 & 0.23 & 5.6 & 0.8 & 15 & 0.28 & 0.54 \\
\hline G-18 & $27 \mathrm{R}-1,28-32$ & 829.90 & 3.5 & 15000 & 7.44 & 0.920 & 205.7 & 1.08 & 157 & 2.9 & 4.91 & 19.39 & 98 & 0.71 & 62.6 & 4.8 & 87 & 23.4 & 4.35 \\
\hline$G-20$ & $28 \mathrm{R}-1,10-14$ & 839.02 & 3.0 & 35000 & & & 116.0 & 0.69 & 89 & 3.6 & 1.71 & 10.64 & 190 & 0.00 & 28.7 & 2.1 & 45 & 15.4 & 1.18 \\
\hline$G-22$ & $29 \mathrm{R}-1,4-8$ & 848.46 & 4.0 & 25000 & 7.44 & 0.945 & 215.6 & 1.56 & 167 & 5.1 & 8.16 & 15.52 & 76 & 1.88 & 53.3 & 3.4 & 103 & & 3.88 \\
\hline G-23 & $31 \mathrm{R}-1,5-7$ & 868.56 & 4.5 & 30000 & 7.28 & 1.171 & 201.4 & 0.95 & 156 & & 5.09 & 17.45 & 68 & 1.65 & 57.1 & 3.2 & 93 & 10.8 & 3.97 \\
\hline G-24 & $34 \mathrm{R}-1,5-7$ & 897.56 & 3.0 & 35000 & & & 138.8 & 0.16 & 111 & & 2.69 & 10.10 & 62 & 0.71 & 31.7 & 1.4 & 30 & 1.27 & 1.71 \\
\hline G-25 & $35 \mathrm{R}-1,5-10$ & 907.28 & 3.0 & 35000 & & & 64.1 & 0.05 & 50 & & 0.67 & 5.32 & 76 & 0.23 & 9.3 & 0.3 & 13 & 0.47 & 0.96 \\
\hline G-26 & $37 \mathrm{R}-1,36-37$ & 926.87 & 3.2 & 35000 & & & 187.1 & 0.76 & 148 & & 3.76 & 15.10 & 76 & 1.19 & 45.4 & 1.2 & 60 & 5.06 & 2.24 \\
\hline
\end{tabular}

Notes: Na values are calculated. In calculating Na, alkalinity is assumed to be $0.5 \mathrm{meg} / \mathrm{kg}$ and $\mathrm{K}$ is assumed to be $3 \mathrm{mmol} / \mathrm{kg}$ if they were not measured. Mg and Ca have been corrected using the equations of Gieskes and Peretsman (1986). 
Table 2. The composition of water extracted from lithified sediment and hydrothermally altered basalt from Site 858 using the GRIND technique.

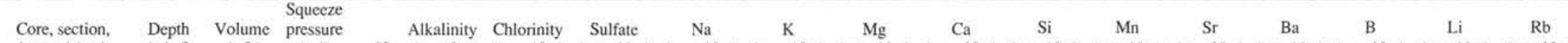

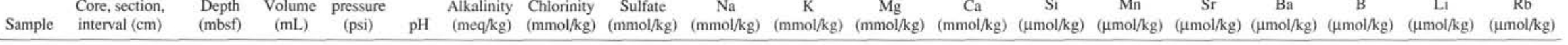

\begin{tabular}{|c|c|c|c|c|c|c|c|c|c|c|c|c|c|c|c|c|c|c|c|}
\hline & $139-858 \mathrm{C}-$ & & 17 & & & & & & & & & & & & & & & & \\
\hline $\begin{array}{l}\text { G-10 } \\
\text { G-11 }\end{array}$ & $\begin{array}{l}10 \mathrm{X}-\mathrm{CC}, 30-35 \\
11 \mathrm{R}-10-10\end{array}$ & $\begin{array}{l}49.63 \\
54.55\end{array}$ & 5.2 & & 7.58 & 1180 & 278.1 & $\begin{array}{l}19.070 \\
1060\end{array}$ & $\begin{array}{l}239.0 \\
2073\end{array}$ & $\begin{array}{l}7.9 \\
6.8\end{array}$ & 22.10 & 12.67 & 72 & $\begin{array}{r}6.22 \\
31.08\end{array}$ & 149 & 0.87 & $\begin{array}{r}84.5 \\
1742\end{array}$ & 344 & 12 \\
\hline G-6 & $12 X-1,131-145$ & 65.38 & 6.0 & & 7.58 & 1.302 & 369.5 & $\begin{array}{r}10.092 \\
8.522\end{array}$ & 312.4 & $\begin{array}{l}0.8 \\
6.2\end{array}$ & 25.20 & $\begin{array}{l}7.43 \\
7.60\end{array}$ & 305 & $\begin{array}{r}31.08 \\
6.78\end{array}$ & $\begin{array}{l}210 \\
273\end{array}$ & $\begin{array}{l}2.29 \\
2.83\end{array}$ & $\begin{array}{l}17.2 \\
214.8\end{array}$ & $\begin{array}{r}54.4 \\
106.0\end{array}$ & 1.2 \\
\hline G-5 & $\begin{array}{l}139-858 \mathrm{D}- \\
6 \mathrm{X}-1,96-105\end{array}$ & 29.81 & 5.0 & & 7.55 & 1.364 & 362.7 & 1.675 & 244.6 & 11.6 & 8.55 & 46.47 & 253 & 12.04 & 554 & 19.25 & & 23.1 & 8.3 \\
\hline G-8 & $\begin{array}{c}139-858 \mathrm{~F}- \\
7 \mathrm{R}-\mathrm{CC}\end{array}$ & 75.10 & 0.5 & & & & 94.3 & 0.153 & & & & & & & & & & & \\
\hline $\mathrm{G}-13$ & 9R-CC, 3-5 & 94.24 & 2.2 & & & & 295.6 & 1.721 & 211.3 & 8.0 & 9.73 & 30.01 & 48 & 2.33 & 357 & 2.77 & 159.7 & 91.6 & 6.5 \\
\hline G-9 & $11 \mathrm{R}-1,0-3$ & 113.62 & 7.0 & & 7.76 & 2.376 & 360.8 & 4.366 & 263.5 & 9.2 & 13.53 & 35.47 & 356 & 14.65 & 510 & 6.95 & 595.6 & 193.8 & 8.7 \\
\hline G-4 & 13R-CC, $0-2$ & 132.91 & 2.3 & & & & 172.1 & 22.456 & 111.5 & 3.1 & 6.12 & 45.00 & 75 & 2.22 & 309 & 0.88 & 113.7 & 63.5 & \\
\hline G-12 & $18 \mathrm{R}-1,65-72$ & 181.99 & 4.2 & & 7.78 & 0.720 & 295.6 & 1.169 & 208.3 & 8.1 & 8.48 & 32.21 & 72 & 0.00 & 399 & 7.67 & 240.9 & 117.6 & 9.4 \\
\hline G-2 & $19 \mathrm{R}-1,9-12$ & 191.01 & 4.6 & & & & 307.3 & 2.056 & 212.8 & 11.6 & 8.92 & 34.29 & 164 & 3.66 & 442 & 13.00 & 397.1 & 164.2 & \\
\hline G-15 & 2 IR-1,9-13 & 210.41 & 4.0 & & 7.45 & 0.832 & 318.9 & 1.525 & 217.2 & 12.1 & 7.28 & 38.92 & 190 & 1.81 & 462 & 15.20 & 401.6 & 179.9 & 5.8 \\
\hline G-7 & $25 \mathrm{R}-1,2-8$ & 248.95 & 2.0 & & & & 311.2 & 1.925 & 217.8 & 10.0 & 7.79 & 35.48 & 232 & 4.26 & 470 & 15.84 & 462.0 & 198.2 & \\
\hline G-16 & $27 \mathrm{R}-1$ (middle) & 267.80 & 2.5 & & & & 65.3 & 1.350 & & 10.2 & & 8.60 & 137 & 1.54 & 68 & 0.63 & 19.1 & 6.9 & 1.4 \\
\hline G-17 & $28 \mathrm{R}-1,28-32$ & 277.80 & 1.5 & & & & 53.8 & 0.355 & & 1.3 & & & 173 & & 70 & 8.88 & & & \\
\hline G-14 & $29 \mathrm{R}-1,85-87$ & 288.06 & 2.0 & & & & 68.4 & 3.594 & & 2.2 & & & 158 & & 55 & 0.69 & & 7.8 & \\
\hline G-1 & $\begin{array}{l}139-858 \mathrm{G}- \\
2 \mathrm{R}-1-56-58\end{array}$ & 287.07 & 23 & 30.000 & & & 38.4 & 0.338 & 35.5 & & 0.23 & 230 & 197 & 0.50 & 20 & 0.37 & 23.5 & 23 & 0.4 \\
\hline G-2 & $5 \mathrm{R}-1,15-18$ & 315.57 & 2.5 & 3,000 & & & 83.7 & 0.195 & 66.9 & & 1.42 & 7.82 & 91 & 0.53 & 62 & 0.92 & 30.7 & 4.8 & 1.4 \\
\hline G-3 & $7 \mathrm{R}-1,55-58$ & 335.37 & 2.8 & 3,000 & & & 57.3 & 0.294 & 48.0 & & 0.70 & 4.97 & 152 & 0.76 & 34 & 0.50 & 28.3 & 2.9 & 0.7 \\
\hline$G-4$ & 10R-1, 90-91 & 365.81 & 2.5 & 3,000 & & & 82.1 & 0.412 & 65.1 & & 1.31 & 8.28 & 109 & 0.62 & 56 & 0.65 & 30.8 & 7.3 & 1.2 \\
\hline G-5 & $12 \mathrm{R}-1,12-15$ & 384.34 & 2.8 & 35,000 & & & 61.5 & 0.305 & 51.1 & & 1.00 & 5.22 & 133 & 0.00 & 38 & 0.42 & 23.9 & 4.2 & 0.8 \\
\hline G-6 & $15 \mathrm{R}-1,20-24$ & 413.52 & 2.2 & 35,000 & & & 75.6 & 0.409 & 61.8 & & 0.79 & 7.21 & 145 & 0.00 & 52 & 0.43 & 25.6 & 6.0 & 1.0 \\
\hline G-7 & $16 \mathrm{R}-1,18-22$ & 423.10 & 2.5 & 35,000 & & & 37.4 & 0.307 & 34.5 & & 0.30 & 2.20 & 271 & 0.00 & 16 & 0.09 & 13.6 & & \\
\hline
\end{tabular}

Notes: Na values are calculated. In calculating Na, alkalinity is assumed to be $0.5 \mathrm{meq} / \mathrm{kq}$ and $\mathrm{K}$ is assumed to be $1 \mathrm{mmol} / \mathrm{kg}$ if they were not measured. Mg and Ca have been corrected using the equations of Gieskes and Peretsman (1986). 


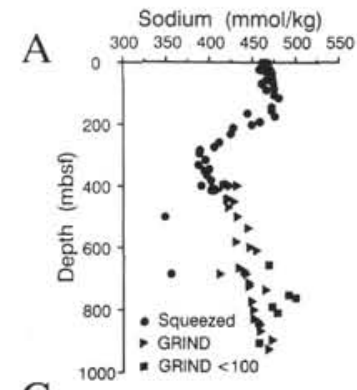

C
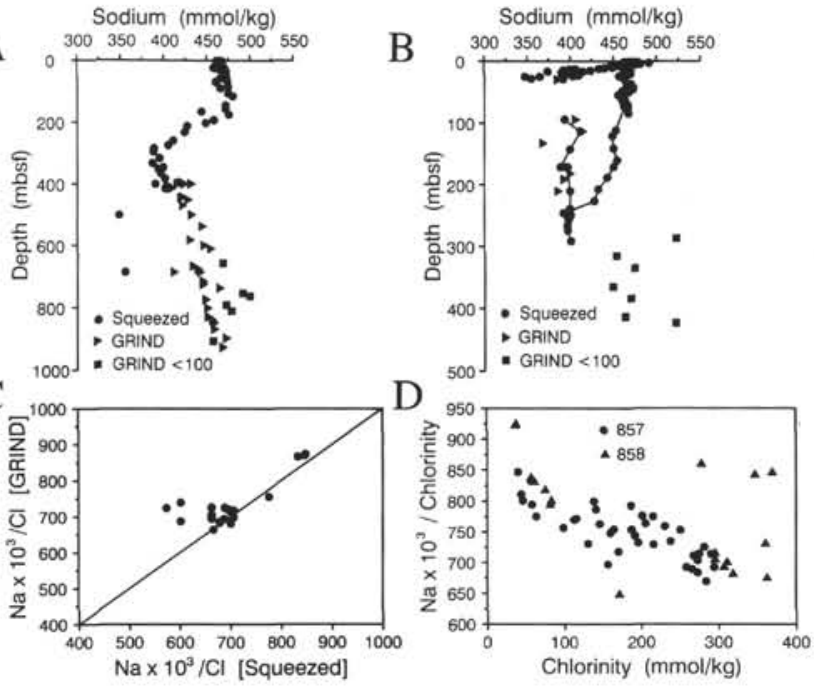

Figure 1. Profiles of Na from squeezed and GRIND samples, including GRIND samples with chlorinity less than $100 \mathrm{mmol} / \mathrm{kg}$. A. Site 857 . GRIND samples have been corrected to a chlorinity of $590 \mathrm{mmol} / \mathrm{kg}$. B. Site 858 . GRIND samples have been corrected to a chlorinity of $565 \mathrm{mmol} / \mathrm{kg}$. Lines connect samples from Holes $858 \mathrm{~A}$ and $858 \mathrm{~F}$. C. Plot of the ratio of Na to chlorinity in GRIND samples vs. squeezed samples. The slope of the line is 1.0. D. The ratio of Na to chlorinity in GRIND samples vs. chlorinity.

present profiles of porosity (Davis, Mottl, Fisher, et al., 1992) at depths where GRIND samples were retrieved along with profiles of chlorinity for all of the GRIND samples. There is an overall decrease in the porosity and chlorinity of the GRIND samples with depth. Superimposed on these decreases are excursions to much lower values, caused by changes in lithology from lithified sediment to hydrothermally altered basalt and gabbro. A good correlation exists between the porosity of the sediment or rock and the resulting chlorinity of the GRIND sample (Fig. 2C), because the volume of distilled water added was generally kept constant at $5 \mathrm{~mL}$. Although the data are consistent with a general increase in $\mathrm{Na}$ with depth, ion exchange also must occur. Samples with low porosity are more affected by ion-exchange processes; thus, the lower chlorinity samples have a higher $\mathrm{Na} / \mathrm{Cl}$ ratio, which results in $\mathrm{Na}$ depth profiles that are not smooth. Smoothed profiles are likely because non-smoothed profiles require diffusive fluxes from lithologic units with high concentrations to those with low concentrations. Based on ion diffusion coefficients that are about 30 times greater at $275^{\circ} \mathrm{C}$ than at $2^{\circ} \mathrm{C}$ (Nigrini, 1970), calculated chemical fluxes resulting from differences in chemical concentrations between lithologic units that are thin, typically less than $10 \mathrm{~m}$ thick, are large enough to impact the concentration in the solid phase. However, the solid phase does not record such fluxes (Goodfellow and Peter, this volume). Therefore, although $\mathrm{Na}$ increases with depth, ion exchange caused by dilution during the GRIND process affects the concentration of dissolved Na. Despite the above-mentioned problems, the GRIND technique is useful for obtaining pore-water $\mathrm{Na}$ data from samples that yield waters with a chlorinity $>100 \mathrm{mmol} / \mathrm{kg}$.

Similar profiles and plots for the other major monovalent $(\mathrm{K})$ and divalent cations $(\mathrm{Mg}$ and $\mathrm{Ca}$ ) are presented in Figures 3, 4, and 5, respectively. As was the case for $\mathrm{Na}$, concentrations of $\mathrm{K}$ are generally higher in GRIND samples than squeezed samples (Fig. 3C), but there is no obvious effect of ion exchange in the low-chlorinity $(<100$ $\mathrm{mmol} / \mathrm{kg}$ ) samples (Fig. 3D). In contrast to the $\mathrm{Na}$ and $\mathrm{K}$ data, concentrations of $\mathrm{Mg}$ and $\mathrm{Ca}$ are typically greater in the squeezed samples than in the GRIND samples (Figs. 4C and 5C). Ion exchange in the low-chlorinity GRIND samples is clearly evident in the $\mathrm{Mg}$ data but less apparent in the Ca data (Figs. 4D and 5D). Some anomalously high concentrations of $\mathrm{Ca}$, in both the squeezed and GRIND samples,

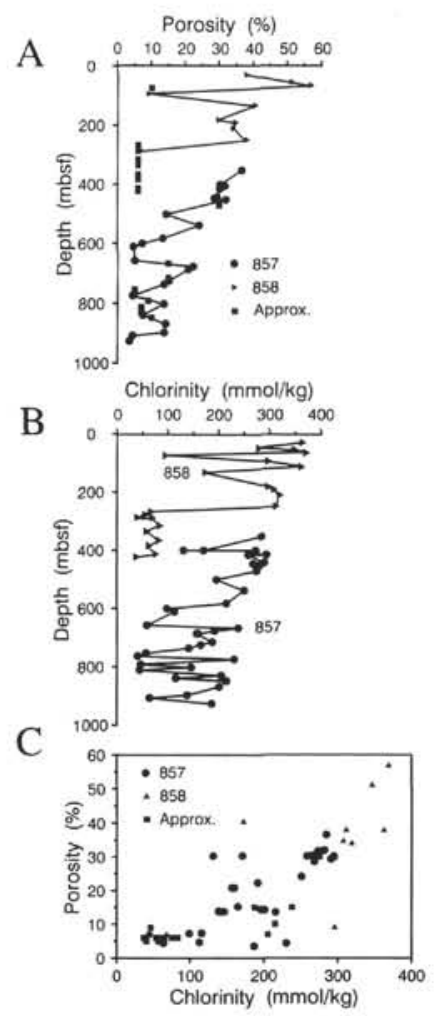

Figure 2. Profiles of porosity and chlorinity of GRIND samples. For GRIND samples that do not have porosity values, we approximated the porosity from neighboring samples (Davis, Mottl, Fisher, et al., 1992). Also shown is a plot of porosity vs, chlorinity.

Table 3. GRIND samples that have a corresponding squeezed aliquot.

\begin{tabular}{lll}
\hline Sample & Hole & $\begin{array}{c}\text { Core, section, } \\
\text { interval }(\mathrm{cm})\end{array}$ \\
\hline G-5 & $857 \mathrm{C}$ & $37 \mathrm{R}-1,140-150$ \\
G-2 & $857 \mathrm{C}$ & $46 \mathrm{R}-2,0-15$ \\
G-1 & $857 \mathrm{C}$ & $46 \mathrm{R}-2,0-15$ \\
G-3 & $857 \mathrm{C}$ & $46 \mathrm{R}-2,0-15$ \\
G-4 & $857 \mathrm{C}$ & $48 \mathrm{R}-1,132-147$ \\
G-6 & $857 \mathrm{C}$ & $49 \mathrm{R}-1135-150$ \\
G-21 & $857 \mathrm{C}$ & $49 \mathrm{R}-1,135-150$ \\
G-19 & $857 \mathrm{C}$ & $62 \mathrm{R}-1,142-150$ \\
G-5 & $857 \mathrm{D}$ & $12 \mathrm{R}-1,16-20$ \\
G-7 & $857 \mathrm{D}$ & $12 \mathrm{R}-1,16-20$ \\
G-10 & $858 \mathrm{C}$ & $10 \mathrm{X}-\mathrm{CC}, 30-35$ \\
G-11 & $858 \mathrm{C}$ & $11 \mathrm{R}-1,0-10$ \\
G-6 & $858 \mathrm{C}$ & $12 \mathrm{X}-1,131-145$ \\
G-5 & $858 \mathrm{D}$ & $6 \mathrm{X}-1,96-105$ \\
G-13 & $858 \mathrm{~F}$ & $9 \mathrm{R}-\mathrm{CC}, 3-5$ \\
G-9 & $858 \mathrm{~F}$ & $11 \mathrm{R}-1,0-3$ \\
G-12 & $858 \mathrm{~F}$ & $18 \mathrm{R}-1,65-72$ \\
G-2 & $858 \mathrm{~F}$ & $19 \mathrm{R}-1,9-12$ \\
G-15 & $858 \mathrm{~F}$ & $21 \mathrm{R}-1,9-13$ \\
G-7 & $858 \mathrm{~F}$ & $25 \mathrm{R}-1,2-8$ \\
\hline
\end{tabular}

result from dissolution of carbonates and/or anhydrite during sample handling, based on corresponding increases in the concentrations of alkalinity and sulfate. As with $\mathrm{Na}$, data for $\mathrm{K}, \mathrm{Mg}$, and $\mathrm{Ca}$ from samples with chlorinity greater than $100 \mathrm{mmol} / \mathrm{kg}$ are useful for estimating in-situ concentrations.

Dilution with distilled water, precipitation reactions, and sample handling at room temperature cause GRIND samples to have generally lower concentrations of alkalinity, sulfate, and dissolved silica than squeezed samples (Fig. 6). The difference in the alkalinity prob- 

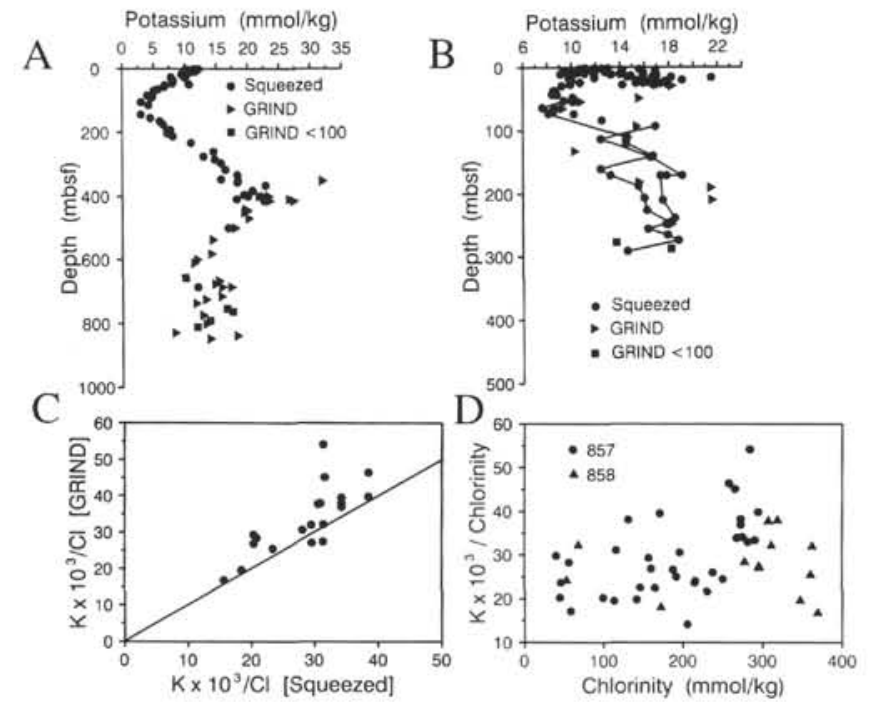

Figure 3. Profiles of $\mathrm{K}$ from squeezed and GRIND samples, including GRIND samples with chlorinity less than $100 \mathrm{mmol} / \mathrm{kg}$. A. Site 857 . GRIND samples have been corrected to a chlorinity of $590 \mathrm{mmol} / \mathrm{kg}$. B. Site 858 . GRIND samples have been corrected to a chlorinity of $565 \mathrm{mmol} / \mathrm{kg}$. Lines connect samples from Holes $858 \mathrm{~A}$ and $858 \mathrm{~F}$. C. Plot of the ratio of $\mathrm{K}$ to chlorinity in GRIND samples vs. squeezed samples. The slope of the line is 1.0. D. The ratio of $\mathrm{K}$ to chlorinity in GRIND samples vs. chlorinity.
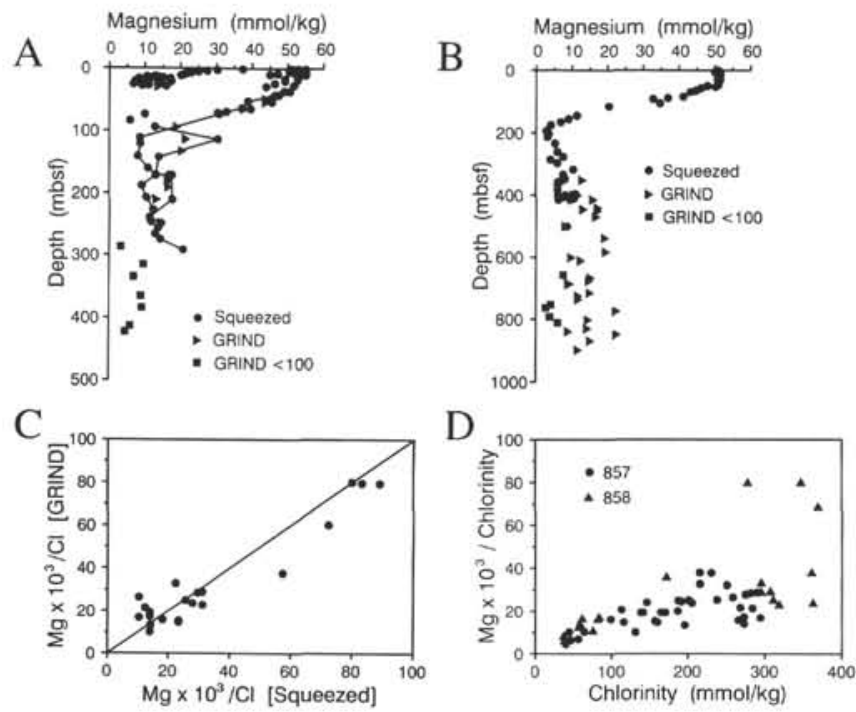

Figure 4. Profiles of $\mathrm{Mg}$ from squeezed and GRIND samples, including GRIND samples with chlorinity less than $100 \mathrm{mmol} / \mathrm{kg}$. A. Site 857 . GRIND samples have been corrected to a chlorinity of $590 \mathrm{mmol} / \mathrm{kg}$. B. Site 858 . GRIND samples have been corrected to a chlorinity of $565 \mathrm{mmol} / \mathrm{kg}$. Lines connect samples from Holes $858 \mathrm{~A}$ and $858 \mathrm{~F}$. C. Plot of the ratio of $\mathrm{Mg}$ to chlorinity in GRIND samples vs. squeezed samples. The slope of the line is 1.0. D. The ratio of $\mathrm{Mg}$ to chlorinity in GRIND samples vs. chlorinity.

ably results from differential rates of carbonate precipitation and dissolution during sample handling. The most dilute GRIND samples $(<200 \mathrm{mmol} \mathrm{Cl} / \mathrm{kg}$ ) have higher alkalinities (Fig. 6B), suggesting that these samples are either better preserved or are more affected by dissolution of calcium carbonate. Likewise, the lower concentrations of sulfate probably result from differences in the rate of inorganic precipitation-dissolution reactions. Cranston (1991) found that dilution may lower the concentration of sulfate, but he did not attribute
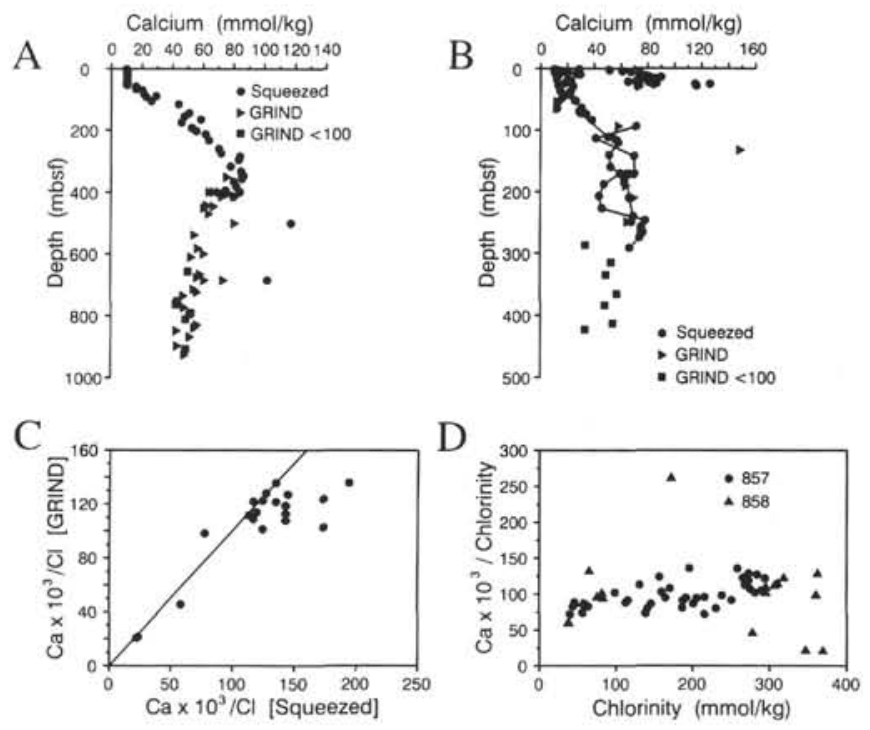

Figure 5. Profiles of $\mathrm{Ca}$ from squeezed and GRIND samples, including GRIND samples with chlorinity less than $100 \mathrm{mmol} / \mathrm{kg}$. A. Site 857 . GRIND samples have been corrected to a chlorinity of $590 \mathrm{mmol} / \mathrm{kg}$. B. Site 858 . GRIND samples have been corrected to a chlorinity of $565 \mathrm{mmol} / \mathrm{kg}$. Lines connect samples from Holes $858 \mathrm{~A}$ and $858 \mathrm{~F}$. C. Plot of the ratio of $\mathrm{Ca}$ to chlorinity in GRIND samples vs. squeezed samples. The slope of the line is 1.0. D. The ratio of $\mathrm{Ca}$ to chlorinity in GRIND samples vs. chlorinity.

this to a specific reaction or exchange process. Anomalously high ratios of sulfate to chlorinity result from dissolution of calcium sulfate during sample handling and processing, as documented by the Shipboard Scientific Party (1992). The presence of sulfate in these samples may reflect contamination with seawater introduced through fractures created during drilling. Based on the discussion above, both the alkalinity and sulfate data obtained using the GRIND technique are suspect. Cranston (1991) found that concentrations of dissolved silica increased in diluted samples, in contrast to our data (Fig. 6E). This difference probably reflects the in-situ and processing temperatures. All of the pore-water GRIND samples from Leg 139 were taken at depths where the temperature exceeds $50^{\circ} \mathrm{C}$, about the temperature at which sampling artifacts lower the concentration of dissolved silica below in-situ values (Wheat and Tribble, this volume). Thus, measurements of dissolved silica in pore waters from Leg 139 obtained by both squeezed and GRIND techniques are not representative of in-situ values and will not be discussed further.

\section{Minor and Trace Ions}

At Site 857 the general trend between GRIND and squeezed concentrations of $\mathrm{Sr}$ is similar to that for $\mathrm{Mg}$ (Figs. 3C and $7 \mathrm{C}$ ). Dilution during the GRIND technique causes ion exchange which removes $\mathrm{Sr}$ from pore waters at Site 857 (Fig. 7D). The greatest effect is observed in GRIND samples from Site 857 with chlorinity less than about $100 \mathrm{mmol} / \mathrm{kg}$ (Fig. 7D). Concentrations of Sr in GRIND samples from Site 858 are much greater than those in squeezed samples (Fig. 7C). The ratio of $\mathrm{Sr}$ from GRIND samples to $\mathrm{Sr}$ in squeezed samples is about 3.8 for Site 858 compared to less than 1 for Site 857 . These high GRIND values must be caused by dissolution reactions during the GRIND technique. Although the dissolution reactions are unknown, differences between Sites 857 and 858 could be a result of the Sr-bearing minerals at each site. Even though sediment or rock from Site 857 contains about twice as much $\mathrm{Sr}$ than sediment or rock from Site 858 (Goodfellow and Peter, this volume), the Sr at Site 858 is probably in secondary minerals that readily dissolve during dilution, whereas $\mathrm{Sr}$ at Site 857 is probably in primary minerals that are more resistant to dissolution. Ion exchange in samples from Site 858 

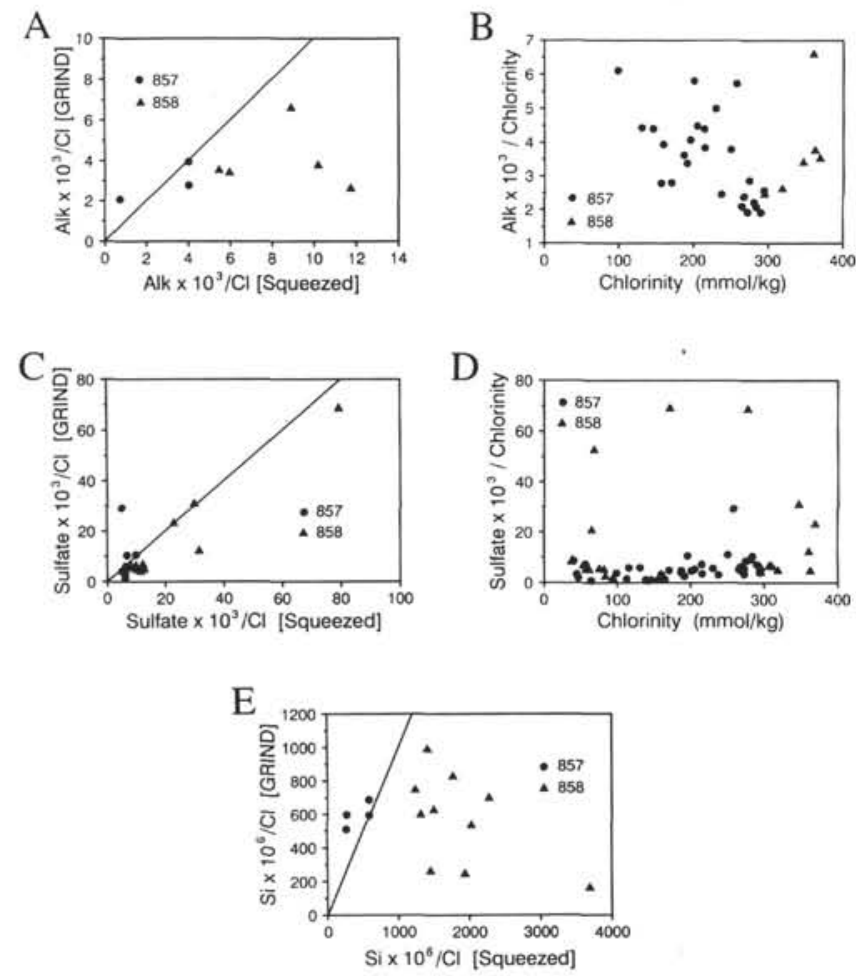

Figure 6. Plots of the alkalinity (A), sulfate $(\mathbf{C})$, and dissolved silica $(\mathbf{E})$ to chlorinity ratio of GRIND samples vs. squeezed samples. The slopes of the lines are 1.0. The ratio of alkalinity (B) and sulfate (D) to chlorinity in GRIND samples vs. chlorinity.

with chlorinity less than $100 \mathrm{mmol} / \mathrm{kg}$ may lower $\mathrm{Sr}$ concentration in the GRIND sample (Fig. 7E).

Concentrations of dissolved $\mathrm{Ba}$ in GRIND samples are at least 2.4 times greater than those in squeezed samples (Fig. 8). The high and scattered concentrations of $\mathrm{Ba}$ in the GRIND samples from Site 857 probably reflect desorption from surfaces during the dilution phase of the GRIND technique (Fig. 8C). Ba concentrations measured in GRIND samples from Hole $858 \mathrm{G}$ approach the limits of detection in these low-chlorinity samples, implying that contamination is minimal. Dilution does not appear to remove Ba systematically from samples with chlorinity less than about $100 \mathrm{mmol} / \mathrm{kg}$ (Fig. 8D), in contrast to the $\mathrm{Mg}$ and $\mathrm{Sr}$ data. Based on the large difference between the GRIND and squeezed samples and the observed scatter, the concentrations of $\mathrm{Ba}$ in pore waters obtained using the GRIND technique are suspect.

Most concentrations of dissolved Mn in GRIND samples are less than the values measured in squeezed samples (Fig. 9). This decrease is probably caused by oxidation during grinding and squeezing as oxygen is introduced by exchange with the atmosphere and in the deionized distilled water. Oxidized $\mathrm{Mn}$ is very insoluble relative to reduced $\mathrm{Mn}$; thus, oxidation of dissolved $\mathrm{Mn}$ lowers the concentration in the GRIND pore-water samples. The most diluted GRIND samples $(<100 \mathrm{mmol} \mathrm{Cl} / \mathrm{kg})$ from Site 857 have lower Mn concentrations than neighboring samples (Fig. 9D), implying that ion-exchange processes may influence the concentration of $\mathrm{Mn}$, or that oxidation reactions are accelerated in the more dilute pore-water samples which have greater concentrations of dissolved oxygen than samples that are less diluted. In either case the Mn data are suspect.

Concentrations of dissolved Rb in GRIND samples are generally higher than values measured in squeezed samples, as was the case for $\mathrm{Na}$ (Figs. 1 and 10). Unlike the Na data, concentrations of the monovalent cation $\mathrm{Rb}$ do not increase in samples with chlorinity less than about $100 \mathrm{mmol} / \mathrm{kg}$, and yet concentrations of Rb may actually be less
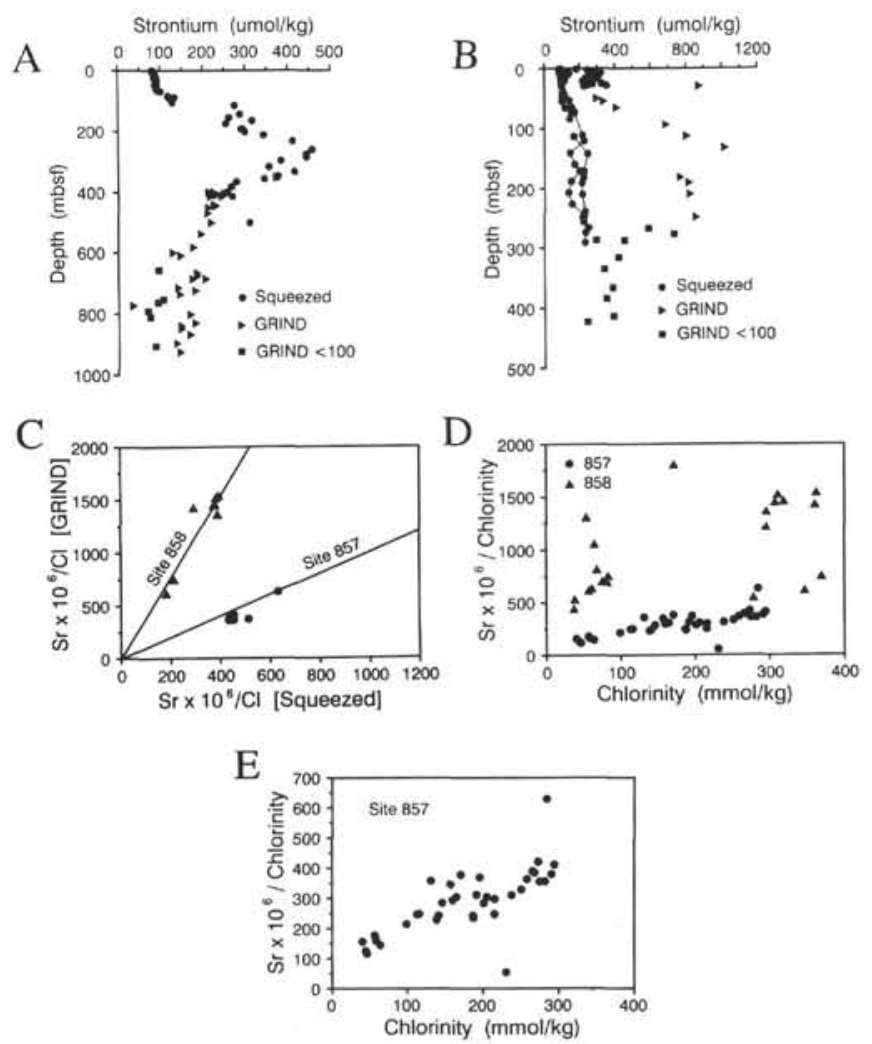

Figure 7. Profiles of $\mathrm{Sr}$ from squeezed and GRIND samples, including GRIND samples with chlorinity less than $100 \mathrm{mmol} / \mathrm{kg}$. A. Site 857 . GRIND samples have been corrected to a chlorinity of $590 \mathrm{mmol} / \mathrm{kg}$. B. Site 858 . GRIND samples have been corrected to a chlorinity of $565 \mathrm{mmol} / \mathrm{kg}$. Lines connect samples from Holes 858A and 858F. C. Plot of the ratio of $\mathrm{Sr}$ to chlorinity in GRIND samples vs. squeezed samples. The slopes of the lines are 1.0 and 3.8 . D. The ratio of $\mathrm{Sr}$ to chlorinity in GRIND samples vs. chlorinity.

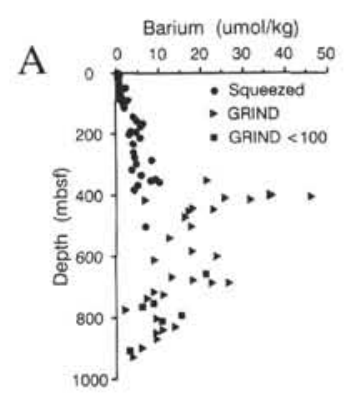

C

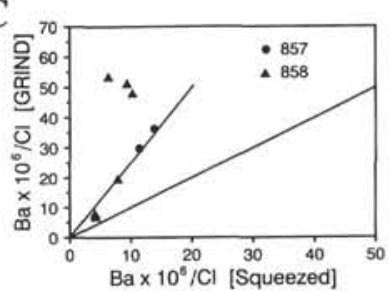

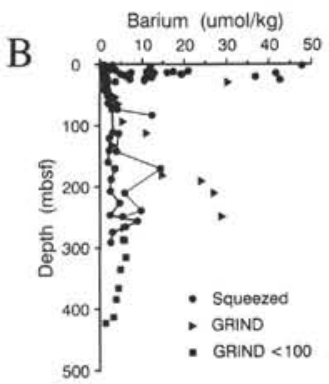

$\mathrm{D}$

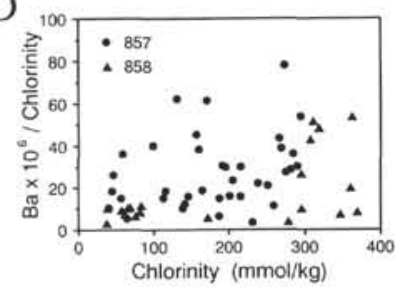

Figure 8. Profiles of Ba from squeezed and GRIND samples, including GRIND samples with chlorinity less than $100 \mathrm{mmol} / \mathrm{kg}$. A. Site 857 . GRIND samples have been corrected to a chlorinity of $590 \mathrm{mmol} / \mathrm{kg}$. B. Site 858 . GRIND samples have been corrected to a chlorinity of $565 \mathrm{mmol} / \mathrm{kg}$. Lines connect samples from Holes $858 \mathrm{~A}$ and $858 \mathrm{~F}$. C. Plot of the ratio of Ba to chlorinity in GRIND samples vs. squeezed samples. The slopes of the lines are 1.0 and 2.4. D. The ratio of Ba to chlorinity in GRIND samples vs. chlorinity. 

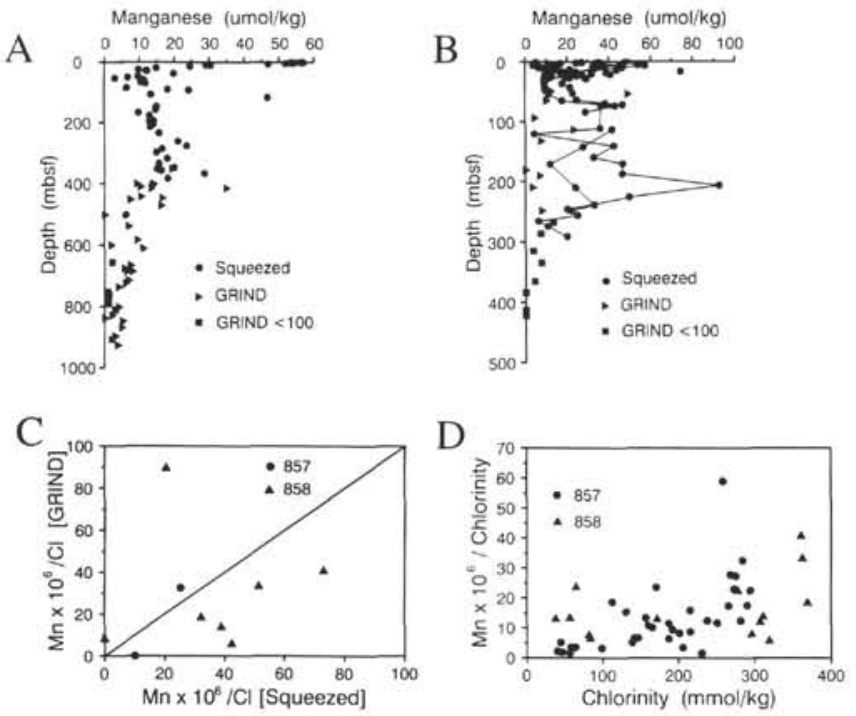

Figure 9. Profiles of Mn from squeezed and GRIND samples, including GRIND samples with chlorinity less than $100 \mathrm{mmol} / \mathrm{kg}$. A. Site 857 . GRIND samples have been corrected to a chlorinity of $590 \mathrm{mmol} / \mathrm{kg}$. B. Site 858 . GRIND samples have been corrected to a chlorinity of $565 \mathrm{mmol} / \mathrm{kg}$. Lines connect samples from Holes $858 \mathrm{~A}$ and $858 \mathrm{~F}$. C. The ratio of $\mathrm{Mn}$ to chlorinity in GRIND samples vs. squeezed samples. The slope of the line is 1.0. D. The ratio of Mn to chlorinity in GRIND samples vs. chlorinity.
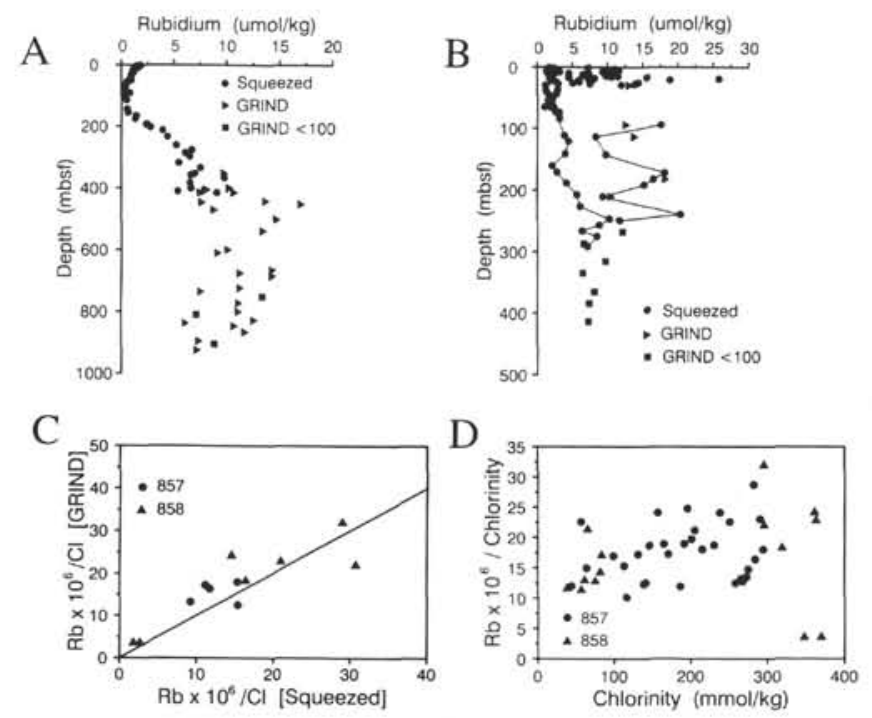

Figure 10. Profiles of $\mathrm{Rb}$ from squeezed and GRIND samples, including GRIND samples with chlorinity less than $100 \mathrm{mmol} / \mathrm{kg}$. A. Site 857 . GRIND samples have been corrected to a chlorinity of $590 \mathrm{mmol} / \mathrm{kg}$. B. Site 858 . GRIND samples have been corrected to a chlorinity of $565 \mathrm{mmol} / \mathrm{kg}$. Lines connect samples from Holes $858 \mathrm{~A}$ and $858 \mathrm{~F}$. C. The ratio of $\mathrm{Rb}$ to chlorinity in GRIND samples vs. squeezed samples. The slope of the line is 1.0. D. The ratio of $\mathrm{Rb}$ to chlorinity in GRIND samples vs. chlorinity.

than expected in samples with chlorinity less than about $100 \mathrm{mmol} / \mathrm{kg}$ (Fig. 10D). Variation in the concentration of Rb in GRIND samples from Site 857 compared with the tight trend observed in the squeezed data illustrates the composite of artifacts that affect concentrations of $\mathrm{Rb}$ in GRIND samples (Figs. 10A and 10B). Although some scatter exists in the profiles (Figs. 10A and 10B), concentrations of $\mathrm{Rb}$ in pore waters from the GRIND technique appear to be representative of the in-situ concentrations.
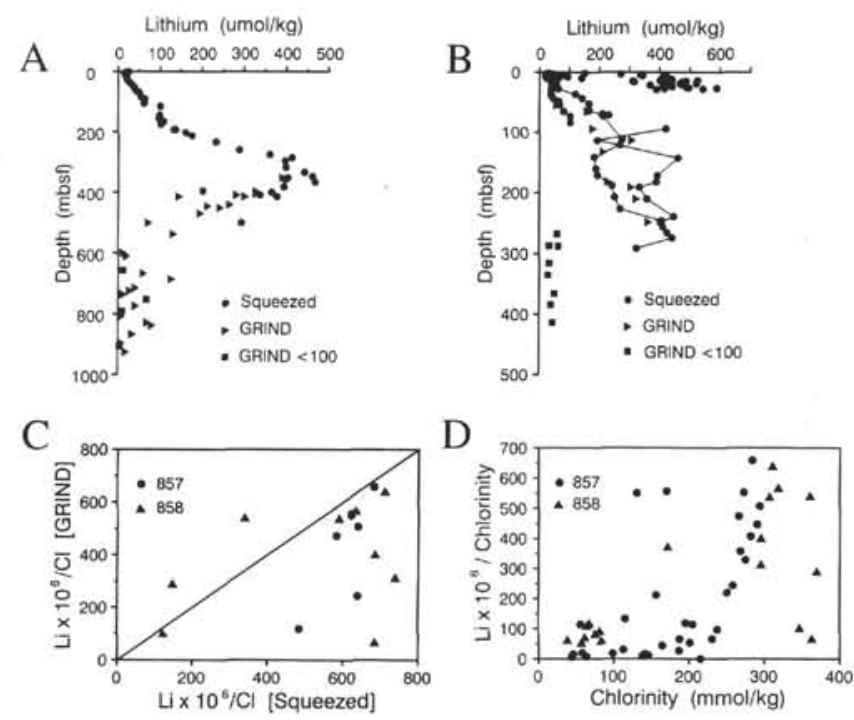

Figure 11. Profiles of $\mathrm{Li}$ from squeezed and GRIND samples, including GRIND samples with chlorinity less than $100 \mathrm{mmol} / \mathrm{kg}$. A. Site 857 . GRIND samples have been corrected to a chlorinity of $590 \mathrm{mmol} / \mathrm{kg}$. B. Site 858 . GRIND samples have been corrected to a chlorinity of $565 \mathrm{mmol} / \mathrm{kg}$. Lines connect samples from Holes $858 \mathrm{~A}$ and $858 \mathrm{~F}$. C. The ratio of $\mathrm{Li}$ to chlorinity in GRIND samples vs. squeezed samples. The slope of the line is 1.0. D. The ratio of Li to chlorinity in GRIND samples vs. chlorinity.

In contrast to $\mathrm{Na}, \mathrm{K}$, and $\mathrm{Rb}$, concentrations of dissolved $\mathrm{Li}$ in GRIND samples are generally less than the values measured in squeezed samples (Fig. 11). Either (1) dilution of GRIND samples to a final sample chlorinity that is below about $250 \mathrm{mmol} / \mathrm{kg}$ results in reactions that remove about $75 \%$ of the dissolved $\mathrm{Li}$ from the sample, or (2) concentrations of $\mathrm{Li}$ in samples retrieved below the first sill in Site 857 ( $471 \mathrm{mbsf}$ ) and below the start of lithologic Unit V (mafic extrusive rock) in Holes $858 \mathrm{~F}$ and $858 \mathrm{G}$ ( $267 \mathrm{mbsf}$ ) are generally lower than concentrations in the cooler overlying sediment. Because the $\mathrm{Li}$ data are unlike the $\mathrm{Na}, \mathrm{K}$, and $\mathrm{Rb}$ data and because concentrations of $\mathrm{Li}$ generally increase downhole in sediments with intruded sills (Gieskes et al., 1982), we suggest that dilution of pore waters during the GRIND technique greatly impacts the concentration of dissolved $\mathrm{Li}$. This implies the GRIND data for $\mathrm{Li}$ are suspect.

Concentrations of dissolved B are generally lower in GRIND samples than in squeezed samples (Fig. 12). Similar to the Li data, dilution of samples with chlorinity less than about $250 \mathrm{mmol} / \mathrm{kg}$ probably causes the removal of about half of the dissolved B from the sample (Fig. 12D); however, most of the low-chlorinity and B concentrations are in samples retrieved from below the first sill in Site 857 and below the start of lithologic Unit V in Holes 858F and 858G. These are generally lower than concentrations in the cooler overlying sediment. Thus, as for $\mathrm{Li}$, the observed $\mathrm{B} /$ chlorinity vs. chlorinity trend may reflect changes in the in-situ concentration with depth and not artifacts incurred during sample handling. Until one of the two possibilities can be validated, B data should remain suspect.

\section{CONCLUSIONS}

Concentrations of $\mathrm{Mg}, \mathrm{Ca}, \mathrm{Na}, \mathrm{K}$, and $\mathrm{Rb}$ in GRIND samples from Sites 857 and 858 and $\mathrm{Sr}$ in GRIND samples from Site 857 are comparable to values from squeezed samples. These good correlations imply that the GRIND technique is a useful tool for obtaining reliable information on the chemical composition of pore water from indurated and hydrothermally altered sediment and basalt. We have made no attempt to statistically treat these correlations because much of the data are centered about a small concentration range and because at present this technique should only be used to estimate pore-water- 

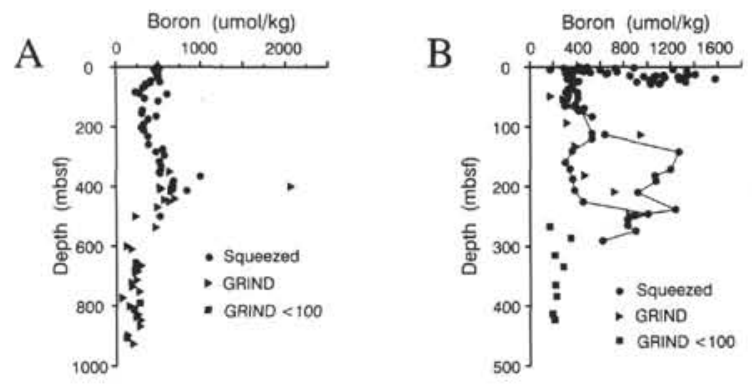

$\mathrm{C}$
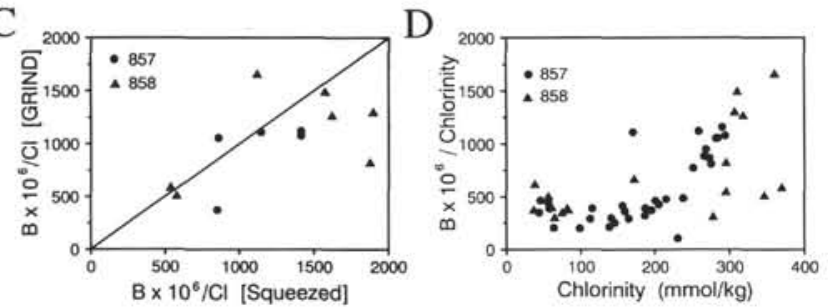

Figure 12. Profiles of $B$ from squeezed and GRIND samples, including GRIND samples with chlorinity less than $100 \mathrm{mmol} / \mathrm{kg}$. A. Site 857 . GRIND samples have been corrected to a chlorinity of $590 \mathrm{mmol} / \mathrm{kg}$. B. Site 858 . GRIND samples have been corrected to a chlorinity of $565 \mathrm{mmol} / \mathrm{kg}$. Lines connect samples from Holes $858 \mathrm{~A}$ and $858 \mathrm{~F}$. C. The ratio of B to chlorinity in GRIND samples vs. squeezed samples. The slope of the line is 1.0. D. The ratio of B to chlorinity in GRIND samples vs. chlorinity.

concentration, until more is known about the effects of different sediment types and in-situ conditions on the chemical reactions that occur during the GRIND procedure. The reproducibility of the GRIND method has been assessed by determining the average standard deviation of the concentration to chlorinity ratio for the one sample that was analyzed in triplicate and the two samples that were analyzed in duplicate. The average standard deviations for $\mathrm{Ca}, \mathrm{Na}, \mathrm{K}$, and $\mathrm{Sr}$ are below $6 \%$. The average standard deviations for $\mathrm{Mg}$ and $\mathrm{Rb}$ are $17 \%$ and $9 \%$, respectively.

In contrast to $\mathrm{Mg}, \mathrm{Ca}, \mathrm{Na}, \mathrm{K}, \mathrm{Sr}$, and $\mathrm{Rb}$, concentrations of alkalinity, sulfate, dissolved silica, $\mathrm{Ba}, \mathrm{Mn}, \mathrm{Li}$, and $\mathrm{B}$ in most GRIND samples do not correlate well with concentrations measured in pore waters from squeezed samples, because of precipitation, dissolution, and ion-exchange reactions that occur during sample processing. The average standard deviations of these analyses on GRIND samples are between $11 \%$ and $26 \%$ for alkalinity, $\mathrm{Si}, \mathrm{Ba}, \mathrm{Mn}, \mathrm{B}$, and $\mathrm{Li}$, and $43 \%$ for sulfate.

The GRIND technique produces the most reliable results when the sample chlorinity is greater than about $100 \mathrm{mmol} / \mathrm{kg}$. Ion exchange in samples with chlorinity lower than $100 \mathrm{mmol} / \mathrm{kg}$ may provide $\mathrm{Na}$ to the pore waters while removing $\mathrm{Mg}, \mathrm{Ca}$, and $\mathrm{Sr}$. Further study is required to confirm this result.

\section{ACKNOWLEDGMENTS}

This research was supported by the Ocean Drilling Program and by a grant from the Joint Oceanographic Institutions U.S. Science Support Program. We would like to thank J. McManus and an anonymous reviewer for their thoughtful reviews of this manuscript. This is contribution number 3533 from the School of Ocean and Earth Science and Technology of the University of Hawaii.

\section{REFERENCES ${ }^{*}$}

Barnes, R.O., 1988. ODP in-situ fluid sampling and measurement: a new wireline tool. In Mascle, A., Moore, J.C., et al., Proc. ODP, Init. Repts., 110: College Station, TX (Ocean Drilling Program), 55-63.

Cranston, R.E., 1991. Testing a higher resolution interstitial-water method for the Ocean Drilling Program. In Barron, J., Larsen, B., et al., Proc. ODP, Sci. Results, 119: College Station, TX (Ocean Drilling Program), 393-400.

Davis, E.E., Mottl, M.J., Fisher, A.T., et al., 1992. Proc. ODP, Init. Repts., 139: College Station, TX (Ocean Drilling Program).

de Lange, G.J., Cranston, R.E., Hydes, D.H., and Boust, D., 1992. Extraction of pore water from marine sediments: a review of possible artifacts with pertinent examples from the North Atlantic. Mar. Geol., 109:53-76.

Gieskes, J.M., Elderfield, H., Lawrence, J.R., Johnson, J., Meyers, B., and Campbell, A., 1982. Geochemistry of interstitial waters and sediments, Leg 64, Gulf of California. In Curray, J.R., Moore, D.G., et al., Init. Repts. DSDP, 64 (Pt. 2): Washington (U.S Govt. Printing Office), 675-694.

Gieskes, J.M., and Peretsman, G., 1986. Water chemistry procedures aboard JOIDES Resolution-some comments. ODP Tech. Note, 5.

Manheim, F.T., and Sayles, F.L., 1974. Composition and origin of interstitial waters of marine sediments based on deep sea drill cores. In Goldberg, E.D. (Ed.), The Sea (Vol. 5): New York (Wiley Interscience), 527-568.

Nigrini, A., 1970. Diffusion in rock alteration systems: I. Prediction of limiting equivalent ionic conductances at elevated temperatures. Am. J. Sci., 269:65-91.

Shipboard Scientific Party, 1992. Explanatory notes. In Davis, E.E., Mottl, M.J., Fisher, A.T., et al., Proc. ODP, Init. Repts., 139: College Station, TX (Ocean Drilling Program), 55-97.

\footnotetext{
Abbreviations for names of organizations and publications in ODP reference lists follow the style given in Chemical Abstracts Service Source Index (published by American Chemical Society).
}

Date of initial receipt: 23 April 1993

Date of acceptance: 24 September 1993

Ms 139SR-234 